\title{
Mutation of Spastin Affects Microtubule Dynamics Through Differential Distribution
}

\author{
Liang Zhi, Cai Zhenbin, Zhang Guowei, Tan Minghui, Lin Hongsheng* \\ Department of Orthopedics, the First Affiliated Hospital of Jinan University, Guangzhou, China \\ Email address: \\ tlinhsh@jnu.edu.cn (Lin Hongsheng) \\ ${ }^{*}$ Corresponding author \\ To cite this article: \\ Liang Zhi, Cai Zhenbin, Zhang Guowei, Tan Minghui, Lin Hongsheng. Mutation of Spastin Affects Microtubule Dynamics Through \\ Differential Distribution. Cell Biology. Vol. 8, No. 2, 2020, pp. 22-26. doi: 10.11648/j.cb.20200802.11
}

Received: July 21, 2020; Accepted: July 29, 2020; Published: August 5, 2020

\begin{abstract}
SPG4 gene encodes Spastin, and its mutations are the main cause of hereditary spastic paraplegia (HSP). There are more than 50 gene mutations in HSP, and mutations of Spastin account for more than $40 \%$, so SPG4 is the key gene that causes the disease. In order to clarify the effect on microtubule stability after inactivation of AAA functional domain caused by mutations of Spastin, we carried out PCR amplification and C413Y site-directed mutation of the target gene according to the sequence of SPG4 in PUBMED gene bank and constructed GFP-Spastin and GFP-Spastin C413Y recombinant plasmids. The recombinant plasmids were introduced into COS7 cells, and the expression of recombinant plasmids in COS7 cells and changes of microtubule stability were observed. The results of colony PCR and gene identification showed that recombinant plasmids were successfully constructed. Western blotting showed that GFP-Spastin and GFP-Spastin C413Y could be expressed normally in COS7 cells. Immunofluorescence assay showed that the distribution of GFP-Spastin was punctate in the cells and GFP-Spastin had strong microtubule cleavage ability, while GFP-Spastin C413Y was almost distributing in the nucleus, and the ability of microtubule cleavage was weakened and the microtubule was in a stable state. Therefore, we concluded that Spastin C413Y changed the distribution of Spastin in cells and weakened the ability of microtubule cleavage. Spastin C413Y accumulated in the nucleus which could not cut microtubules effectively.
\end{abstract}

Keywords: Spastin, Mutation, Microtubule Dynamics, Protein Distribution

\section{Introduction}

SPG4 gene encodes spastin, and its gene mutations are the main cause of hereditary spastic paraplegia (HSP) [1]. HSP is a neurodegenerative disease with axonal degeneration of the bilateral corticospinal tract of the spinal cord, clinical manifestations of increased muscle tone of both lower extremities, hyperreflexia of tendons, positive pathological reflex and scissors-like gait $[2,3]$. There are more than 50 gene mutations in HSP, and Spastin mutations account for more than $40 \%$. It is the key gene that causes the disease, and most of the mutations are located in the AAA region of Spastin [4]. Therefore, expounding the function of the mutations of SPG4 has important scientific research value and clinical significance.

Spastin belongs to the AAA protein family of microtubule cleavage proteins [5]. The three-dimensional structure of human Spastin protein has been analyzed. Spastin contains several main functional regions, including the $\mathrm{N}$-terminal MIT region (microtubuleinteracting and trafficking domain), MBD (microtubule-binding domain), and C-terminal AAA enzyme catalysis. The activated Spastin is usually a circular hexamer, which binds and anchors the microtubules through the MBD region, and segments the microtubules by catalysis of the AAA domain [6]. Spastin is highly conserved in the process of evolution, especially the AAA region, but the AAA region is a high incidence region of mutations in HSP. Mutations in this region lead to changes in the function of Spastin protein [7], resulting in corresponding clinical symptoms in patients with HSP.

Spastin C413 is also located in the AAA region and is a common AAA region mutation in HSP [8]. However, the effect of the Spastin C413Y on microtubule stability is still unclear. Therefore, we constructed Spastin and Spastin C413Y 
eukaryotic expression plasmids and studied their effects on the stability of microtubules in COS7 cells, which provided a model basis for the follow-up study of Spastin cleavage and its internal mechanism.

\section{Materials and Methods}

\subsection{Materials}

COS7 (SCSP-508) cells were purchased from the Cell Bank of the Chinese Academy of Sciences. The types and manufacturers of materials are DMEM medium (GIBCO), fetal bovine serum (BI), cell lysate (Meilunbio), ECL luminescence reagent (Meilunbio), Triton (Meilunbio), 4\% Paraformaldehyde (Meilunbio), mouse antibody GFP polyclonal antibody (SANTA), rat anti-tubulin monoclonal antibody (ABCAM), Alexa Fluor 647 donkey anti-rat (ABCAM), DAPI (EMS) and Lipo2000 (Invitrogen). EcoRI and BGI II restriction enzymes, DNA marker, DNA ligas, and 2x TaqPlus Master Mix (Takara).

\subsection{Methods}

\subsubsection{Construction of GFP-Spastin, GFP-Spastin C413Y Recombinant Plasmids}

The recombinant plasmids GFP-Spastin and GFP-Spastin C413Y were constructed according to the sequence of rat Spastin gene in PUBMED gene bank. The PCR primers of GFP-Spastin and GFP-Spastin C413Y were shown in Table 1. Using rat cDNA as a template, PCR was pre-denatured at $95^{\circ} \mathrm{C}$ for 5 minutes, denatured at $95^{\circ} \mathrm{C}$ for 30 seconds, annealed at $59^{\circ} \mathrm{C}$ for 30 seconds, and extended at $72^{\circ} \mathrm{C}$ for 90 seconds, there were 34 cycles in this process and finally extended for 5 minutes. Then recovered GFP-Spastin and GFP-Spastin C413Y PCR product, the pEGFP-C1 vector was treated with BGIII and EcoRI restriction enzymes. In the presence of DNA ligase, GFP-Spastin and GFP-Spastin $\mathrm{C} 413 \mathrm{Y}$ were ligated at $37^{\circ} \mathrm{C}$ for 30 minutes and transformed into Escherichia coli. The identification of these plasmids was completed by preliminary PCR verification and subsequent sequencing.

Table 1. Primers of constructed plasmids.

\begin{tabular}{lll}
\hline Plasmid & Primer & Sequence \\
\hline \multirow{2}{*}{ GFP-Spastin } & Forward & 5'-GTACCGTCGACTGCAGAATTCATGGCCGCCAAGAGGAGC-3' \\
& Reverse & 5'-AAGTCCGGACTCAGATCTTTAAACAGTGGTGTCTCCAAAG-3' \\
\multirow{2}{*}{ GFP-Spastin C413Y } & Forward & 5'-ATGAAGTTGATAGTCTTTTGTATGAGAGAAGAGAA-3' \\
& Reverse & 5'-TTCTCTTCTCTCATACAAAAGACTATCAACTTCAT-3' \\
\hline
\end{tabular}

\subsubsection{Cultivation and Transfection of $\operatorname{COS} 7$ Cell}

COS7 cells were cultured in DMEM containing $10 \%$ FBS and transferred to 6or 24-well plates. When the cell density reached 80\%-90\%, GFP, GFP-Spastin and GFP-Spastin C413Y were transfected with Lipo2000, and then immunofluorescence and Western blotting analysis was performed.

\subsubsection{Western Blotting}

After COS7 cells were transfected with GFP, GFP-Spastin, GFP-Spastin C413Y recombinant plasmids for 24 hours, then washed with PBS twice, lysed with cell lysate on ice for 10 minutes, and centrifuged at $12000 \mathrm{r} / \mathrm{min}$ for 15 minutes. Collected the supernatant for protein quantification and calculated the protein concentration. The protein was transferred to a $0.45 \mu \mathrm{m}$ PVDF membrane after $10 \%$ SDS-PAGE, blocked with 5\% skimmed milk at room temperature for 1 hour, incubated with the primary anti-GFP antibody at $4{ }^{\circ} \mathrm{C}$ overnight, washed with TBST the next day, and incubated with secondary antibody at room temperature for 1 hour. Finally used ECL to visualize and scan protein bands.

\subsubsection{Immunofluorescence}

After COS7 cells were transfected with plasmids for 24 hours, they were fixed with $4 \%$ paraformaldehyde at $4{ }^{\circ} \mathrm{C}$ for 45 minutes, and then punched twice with Triton for 5 minutes, then blocked with 3\% BSA at room temperature for 1 hour, and incubated the primary antibody overnight at $4^{\circ} \mathrm{C}$. Incubated the fluorescent secondary antibody the next day, and mount the slide after DAPI staining. After the coverslips were dried, the cell immunofluorescence images were acquired by laser confocal scanning, and then the condition of COS7 microtubule cutting was analyzed by Image $\mathrm{J}$ software.

\subsubsection{Statistical Analysis}

The images of COS7 cells were collected by a laser confocal microscope and transmitted to Image $\mathrm{J}$ for processing. Each group was double-blind and repeated 3 times independently. At least 30 COS7 cells were counted in each group, and all data were expressed as mean \pm standard deviation. SPSS23.0 software and one-way ANOVA were used to analyze the correlation.* represented $\mathrm{P}<0.05$, which was considered statistically significant.

\section{Results}

\subsection{The Recombinant Plasmids GFP-Spastin and GFP-Spastin C413Y Were Successfully Constructed}

According to the designed primers, the PCR products of GFP-Spastin and GFP-Spastin C413Y were obtained and ligated to the $\mathrm{pEGFP-C1}$ vector digested by BGI II and EcoR I. Two positive monoclonal cultures were selected from the culture plate and cultured overnight. The plasmid was initially identified by PCR (Figure 1A, B), and sequenced to ensure that the base sequence of the recombinant plasmid was correct (Figure 1C). 

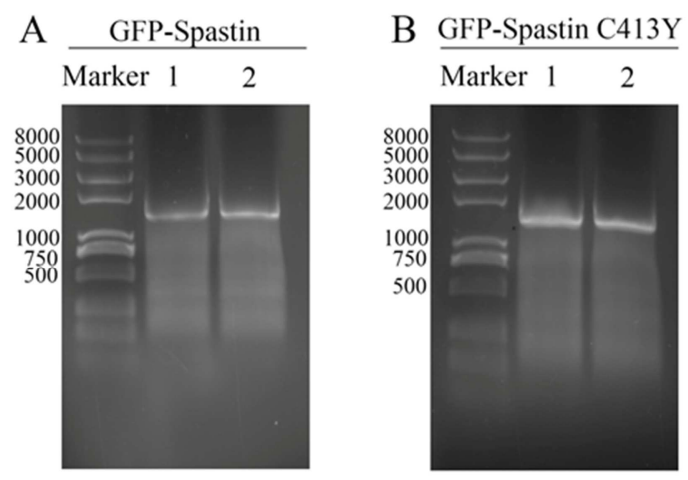

C GFP-Spastin GFP-Spastin C413Y

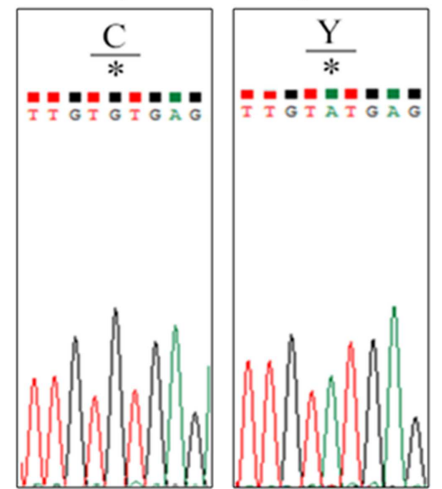

Figure 1. Identification of recombinant plasmids GFP-Spastin and GFP-Spastin C413Y.

A-B. Colony PCR results of GFP-Spastin and GFP-Spastin C413Y recombinant plasmids. Lane 1, DNA marker; Lane 2-3, PCR products of two screened positive monoclonal colonies;

C. Apoint mutation (GFP-Spastin C413Y) in spastin AAA domain replaced cysteine (C) 413 (codon TGT) with tyrosine (Y)(codon TAT)

\subsection{Detection of Protein Expression by Western Blot Assay}

COS7 cells were cultured at a $5 \% \mathrm{CO}_{2} 37^{\circ} \mathrm{C}$ incubator and transfected with GFP, GFP-Spastin and GFP-Spastin C413Y respectively. After 24 hours, the cell lysate was collected for western blot to detect protein expression (Figure 2).

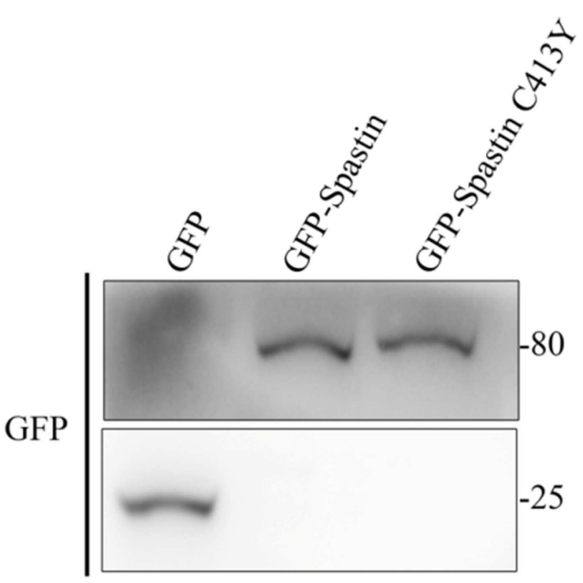

Figure 2. Expression of GFP, GFP-Spastin and GFP-Spastin C413Y.

The molecular weight of GFP is $25 \mathrm{kD}$, and the molecular weight of recombinant plasmids GFP-Spastin and GFP-Spasitn C413Y are both 80kD.

\subsection{The Distribution and Microtubule Cutting Ability of GFP-Spastin and GFP-SpastinC413YAre Significantly Different in COS7 Cells}

In order to observe the distribution and microtubule cutting of GFP, GFP-Spastin and GFP-Spastin C413Y in COS7 cells, they were transfected into COS7 cells respectively and cultured for 24 hours. COS7 cells were observed under a confocal microscope after immunofluorescence staining. GFP distributed uniformly in whole cells and GFP-Spastin distributed in punctate. However, GFP-Spastin C413Y only distributed in nucleus. In addition, GFP-Spastin depolymerized the microtubules of COS7 cells and cut them into small pieces, while the fluorescence intensity of microtubules of COS7 cells in GFP and GFP-Spastin C413Y groups were significantly stronger than that in GFP-Spastin group. The results showed that overexpression of GFP-Spastin had a significant microtubule cleavage effect on COS7 cells, while the Spastin C413Y point mutation weakened the ability to cleave microtubules (Figure 3 ).

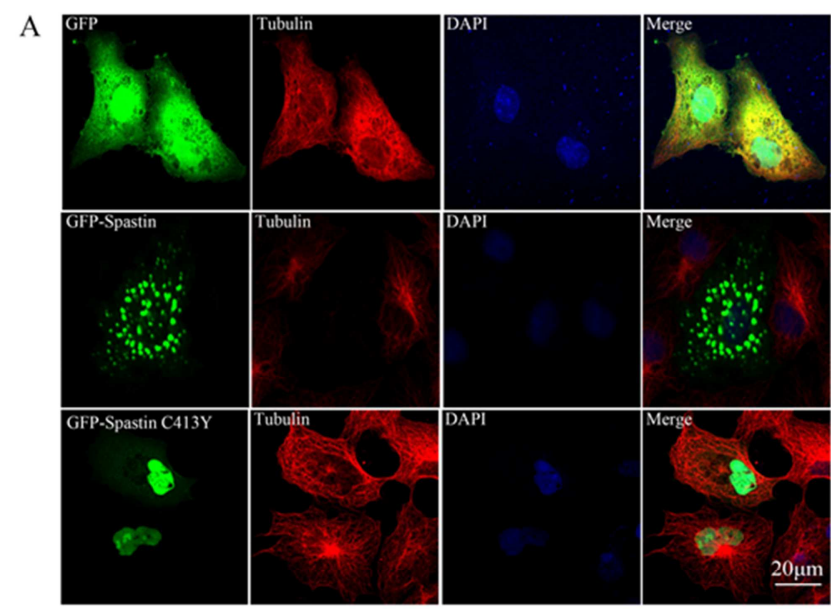

B

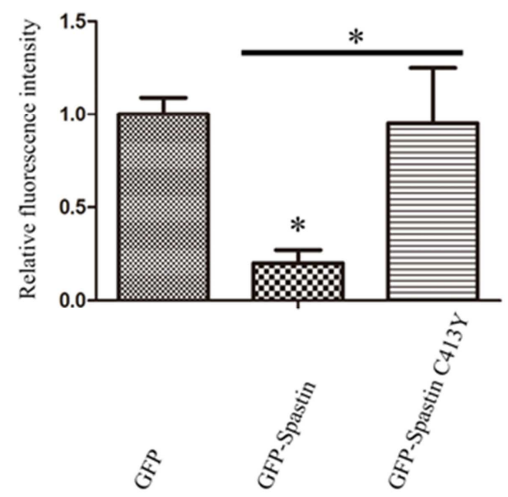

Figure 3. Analysis of overexpression GFP, GFP-Spastin and GFP-Spastin C413Y in COS7 cells by immunofluorescence.

A. GFP (green), GFP-Spastin (green) and GFP-Spastin C413Y (green), Tubulin (red), DAPI (blue) in COS7 cells

B. Quantification of relative fluorescence microtubule intension for the experiment shown in A. Microtubule fluorescence intension in 30 cells were measured per condition. The fluorescence intensity of the control group (GFP) was standardized to 1 . Data represent mean $\pm \mathrm{SD}, \mathrm{P}<0.05$. 


\section{Discussion}

Spastin is a microtubule-cleaving protein involved in a variety of functional regulation processes, including microtubule dynamics [9, 10], membrane transport [11], Cytokinesis [12], neurite growth [13, 14] and axonal transport [15], etc. Spastin plays an important role in neuronal development and participates in the process of neurite outgrowth and formation of lateral branch, which may be related to the regulation of microtubules [16]. When Spastin mutants in drosophila or animals with neuron-specific RNA interference, the stability of microtubules changes [17]. However, the specific mechanism of mutations leading to HSP is not clear. In this study, spastin C413, a common mutation in HSP, was selected as the site to study the function and mechanism of microtubule cleavage.

Spastin monomers are assembled into hexameric cyclic ATP enzymes and cut microtubules along their lengths. Unlike the disassembly mechanism of dynamic instability, the cleavage process is spontaneous and occurs only at the end of microtubules [18]. pastin has the function of cutting microtubules only when it forms a complete hexamer structure. Each Spastin monomer contains a highly conserved AAA ATPase catalytic domain at the carboxyl end and a unique tubulin-binding domain (MBD) [6]. These two domains affect the binding of proteins to microtubule polymers and promote the destruction of ATP hydrolysis-dependent microtubule substrates after examination.

Our study shows that the constructed recombinant plasmid could accurately express Spastin and Spastin C413Y proteins in COS7 cells. The wild type Spastin with dot distribution had strong ability to cut microtubule, while the ability of $\mathrm{C} 413 \mathrm{Y}$ decreased. We speculate that the mutation of $\mathrm{C} 413 \mathrm{Y}$ may lead to the loss of the function of cutting microtubules by changing the ring structure of Spastin monomer hexamer that makes the microtubules stable, which needs to be further verified. In addition, whether other mutations of Spastin have the same experimental phenomenon as $\mathrm{C} 413 \mathrm{Y}$, remain to be further studied. At a deeper level, whether mutations in the AAA region of microtubule cleavage proteins such as Katanin and Fidgetin will produce similar results similar to Spastin C413Y is also a direction of future research on microtubule cleavage proteins. The Spastin C413Y mutation in rats is also one of the mutation points in patients with HSP, which provides a new strategy for the development of rat HSP animal model, and also brings new ideas for the diagnosis and treatment of HSP.

\section{Conclusion}

To sum up, as an important member of the microtubule cutting protein family, the cutting function of Spastin to microtubule depends on its distribution and correct gene expression. Spastin C413Y not only changed the distribution of Spastin in COS7 cells, but also changed the stability of microtubules. The accumulation of Spastin $\mathrm{C} 413 \mathrm{Y}$ in the nucleus could not destroy the cytoskeleton effectively, which changed the ability of wild-type Spastin to cut microtubules.

\section{Acknowledgements}

The authors declare no conflict of interest. This work was supported by the National Natural Science Foundation of China (nos. 31500843 and 37081771331), the Pearl River S\&T Nova Program of Guangzhou (no. 369201806010011), and the Medical Research Foundation of Guangdong Province (A2020308).

\section{References}

[1] Hazan, J., et al., Spastin, a new AAA protein, is altered in the most frequent form of autosomal dominant spastic paraplegia. Nature genetics, 1999. 23 (3): p. 296-303.

[2] McDermott, C. J., et al., Hereditary spastic paraparesis: a review of new developments. Journal of Neurology Neurosurgery And Psychiatry, 2000. 69 (2): p. 150-160.

[3] McDermott, C. J., et al., Clinical features of hereditary spastic paraplegia due to spastin mutation. Neurology, 2006. 67 (1): p. $45-51$.

[4] Fonknechten, N., et al., Spectrum of SPG4 mutations in autosomal dominant spastic paraplegia (vol 9, pg 637, 2000). Human Molecular Genetics, 2005. 14 (3): p. 461-461.

[5] Sharp, D. J. and J. L. Ross, Microtubule-severing enzymes at the cutting edge. Journal Of Cell Science, 2012. 125 (11): p. 2561-2569.

[6] Roll-Mecak, A. and R. D. Vale, Structural basis of microtubule severing by the hereditary spastic paraplegia protein spastin. Nature, 2008. 451 (7176): p. 363-U16.

[7] Shoukier, M., et al., Expansion of mutation spectrum, determination of mutation cluster regions and predictive structural classification of SPAST mutations in hereditary spastic paraplegia (vol 17,pg 187, 2009). European Journal Of Human Genetics, 2009. 17 (3): p. 401-402.

[8] Solowska, J. M., et al., Pathogenic Mutation of Spastin Has Gain-of-Function Effects on Microtubule Dynamics. Journal Of Neuroscience, 2014. 34 (5): p. 1856-1867.

[9] Tadepalle, N., et al., Microtubule-dependent and independent roles of spastin in lipid droplet dispersion and biogenesis. Life science alliance, 2020. 3 (6).

[10] Sandate, C. R., et al., An allosteric network in spastin couples multiple activities required for microtubule severing (vol 26, pg 671, 2019). Nature Structural \& Molecular Biology, 2020. 27 (4): p. $400-400$

[11] Connell, J. W., et al., ESCRT-III-associated proteins and spastin inhibit protrudin-dependent polarised membrane traffic. Cellular and molecular life sciences: CMLS, 2020. 77 (13): p. 2641-2658.

[12] Goyal, U., et al., Spastin-Interacting Protein NA14/SSNA1 Functions in Cytokinesis and Axon Development. Plos One, 2014. 9 (11): p. 11.

[13] Ji, Z. S., et al., Spastin Interacts with CRMP5 to Promote Neurite Outgrowth by Controlling the Microtubule Dynamics. Developmental Neurobiology, 2018.78 (12): p. 1191-1205. 
[14] Jiang, T., et al., PlexinA3 Interacts with CRMP2 to Mediate Sema3A Signalling During Dendritic Growth in Cultured Cerebellar Granule Neurons. Neuroscience, 2020. 434: p. 83-92.

[15] Leo, L., et al., Mutant spastin proteins promote deficits in axonal transport through an isoform-specific mechanism involving casein kinase 2 activation. Human Molecular Genetics, 2017. 26 (12): p. 2321-2334.

[16] Evans, K., et al., Linking axonal degeneration to microtubule remodeling by Spastin-mediated microtubule severing. Molecular Biology Of the Cell, 2004. 15: p. 156A-156A.

[17] Orso, G., et al., Disease-related phenotypes in a Drosphila model of hereditary spastic paraplegia are ameliorated by treatment with vinblastine. Journal Of Clinical Investigation, 2005. 115 (11): p. 3026-3034.

[18] Mitchison, T. and M. Kirschner, Dynamic instability of microtubule growth. Nature, 1984. 312 (5991): p. 237-42. 